Nebenwirkungen unter Statinen

\section{Wie gehen Sie mit Muskelschmerzen um?}

\section{Die Senkung des LDL-Cholesterins mit Statinen zur Primär- und Sekundärprävention verlängert das Leben - wenn die Adhärenz stimmt. Nebenwirkungen beeinträchtigen die Therapietreue, z. B. Muskelschmerzen.}

Eine verschenkte Prävention nannte Prof. Dr. Ulrich Laufs, Homburg/Saar, eine ungenügende Adhärenz zur Statintherapie. Über die Statinnebenwirkung Muskelschmerzen wird immer wieder berichtet, die wissenschaftliche Evidenz dazu ist allerdings begrenzt: Während Beobachtungsstudien auf eine Inzidenz von $10-18 \%$ bei Hochdosis-Statintherapie hinweisen, sind Muskelbeschwerden in placebokontrollierten Studien nicht häufiger als unter Placebo. Trotzdem sollte man die Beschwerden ernst nehmen, rät Laufs. Studien weisen auf eine genetische Disposition hin, und es gibt bei Statintherapie messbare physiologische Veränderungen im Muskel, die einen Effekt nicht ausschließen. Typisch sind:1

- eine symmetrische Lokalisation der Beschwerden,

- ein Auftreten ein bis zwei Wochen nach Beginn der Statineinnahme und

- ein Abklingen zwei bis manchmal vier Wochen nach Absetzen.

- Bei Reexposition treten die Beschwerden häufig wieder auf.

\section{Reexposition nacfh Absetzversuch}

„Wenn die Muskelbeschwerden über zwei bis vier Wochen nach Einnahmebeginn persistieren, sollte man andere Ursachen abklären, z.B. neurologische“, empfahl Laufs. „Das gilt insbesondere bei begleitender CK-Erhöhung.“ Auch Kontraindikationen und mögliche CYP3A4-Interaktionen müssen überprüft werden. Wichtig sei, sich Zeit für die Patienten zu nehmen, betonte Laufs.

Er schlägt im nächsten Schritt das zwei- bis vierwöchige Absetzen und die erneute Reexposition vor. In einer Studie konnten $92 \%$ der Patienten, die wegen Nebenwirkungen die Statintherapie unterbrochen hatten, am Ende weiter eine Statintherapie erhalten - allerdings nicht immer mit demselben Statin und in derselben Dosierung. Laufs empfiehlt, mit sehr niedriger Dosis zu beginnen und einen Statinwechsel zu erwägen. Er empfahl Atorvastatin in einer Anfangsdosis von 2-5 mg. Bei den meisten der ursprünglich statinintoleranten Patienten könne so doch noch eine ausreichende LDL-Senkung mit Statinen erreicht werden. Gelingt das nicht, empfiehlt Laufs die Kombination der maximal tolerierten Statindosis mit Ezetimib. Erst danach sieht Laufs eine Rolle für den neuen PCSK9-Hemmer Alirocumab. Das habe laut Laufs in der ODYSSEY ALTERNATIVE-Studie im Übrigen vergleichbar häufig zu Myalgien geführt wie Atorvastatin und Ezetimib.

Friederike Klein

- Quelle: Symposium Herz und Hirn, auf dem DGN-Kongress,23.-26.9.2015 in Düsseldorf

\section{Hier steht eine Anzeige.}

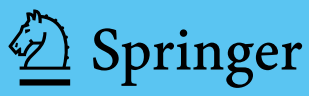

